\title{
Le mot du président...
}

\section{J. Gugenheim (Président du Congrès Soffco-MM 2016)}

(C) Lavoisier SAS 2016

Au sortir d'un hiver et d'un printemps maussades, les beaux jours qui reviennent annoncent le Congrès annuel de la Soffco-MM qui aura lieu à Nice du 23 au 25 juin 2016.

Preuve du succès grandissant de notre société, nous avons reçu 213 abstracts parmi lesquels le comité scientifique de la Soffco-MM a retenu 75 communications orales.

Les pays francophones sont présents avec notamment la Belgique, la Suisse, le Liban, l'Algérie et le Maroc.

Nous avons réuni tous les spécialistes de l'obésité pour faire le point sur les nouveautés scientifiques et cliniques. Comme lors des précédentes éditions, le congrès sera précédé d'une réunion commune avec l'Afero et les CSO. Nos collègues européens seront présents pour faire le point sur la tenue des registres de l'obésité en Europe.

Nous avons fait une place importante à nos partenaires de soins dont l'implication dans la prise en charge de l'obésité est croissante: infirmiers, psychiatres, psychologues, diététiciens, nutritionnistes et kinésithérapeutes...

Vous pourrez, lors des vidéo-forums, faire le point sur les techniques acquises et visualiser les nouvelles interventions chirurgicales. Ce sera également un lieu de rencontre avec nos partenaires de l'industrie qui nous informeront des dernières innovations technologiques. Enfin le samedi, deux séances validantes pour le DPC sont prévues.

J'espère que cette réunion nous permettra non seulement d'actualiser nos connaissances, mais également de mieux nous connaître et de réfléchir ensemble à notre pratique quotidienne.

Je vous souhaite à toutes et à tous un très enrichissant congrès et un séjour agréable sur la Côte d'Azur.

J. Gugenheim $(\bowtie)$

Service de Chirurgie Digestive,

Université de Nice Sophia Antipolis, Hôpital de l'Archet, Nice

e-mail : gugenheim.j@chu-nice.fr 